\title{
Multi-Pair Cable Measurements for 5G Indoor Service Provisioning
}

\author{
Syed Hassan Raza Naqvi*, Pin-Han $\mathrm{Ho}^{\dagger *}$, Sagar Naik, Anwar Haque ${ }^{\ddagger}$, and Tanveer Ahmed ${ }^{\S}$ \\ *Department of Electrical and Computer Engineering, University of Waterloo, ON, Canada. \\ †Zhongtian Broadband Technology Co., Ltd., Nantong, People’s Republic of China \\ ${ }^{\ddagger}$ Department of Computer Science, Western University, ON, Canada \\ $\S_{\text {Nordicity Inc., ON, Canada }}$ \\ E-mails: \{shrnaqvi,p4ho\}@uwaterloo.ca, ahaque32@uwo.ca, tahmed@ nordicity.com
}

\begin{abstract}
This paper considers the 5G new radio (NR) indoor service provisioning scenario where the CPRI link terminates at remote radio unit (RRU) in the building and multi-pair cable, i.e. CAT-5, is used to provide connectivity between distributed antenna unit (DAU) and RRU. The paper focuses on the detailed methodology for multi-pair copper channel measurement including measurement equipment specifications, their respective settings, measurement parameters i.e. characteristics impedance, insertion loss (IL), far-end-crosstalk (FEXT) and near-endcrosstalk (NEXT).

The measurements include the cables for next-generation multipair system, where multi-pair cables, i.e. CAT-5 cables, are used from the RRU to the DAUs in a building. Conventionally, many multi-pair cables share the same duct in a building and interfere with each other, therefore two parallel multi-pair CAT-5 cables of $50 \mathrm{~m}$ each are considered for the measurement scenario. This setup result in 4 twisted pairs terminate at each DAU and this configuration is termed as next-generation multi-pair MIMO (NGMM). The number of twisted pairs is increased by using the Phantom mode circuit connected to the opposite ends of each CAT-5 cable and the resulting configuration is called next-generation multi-pair super MIMO (NGMSM). The cable parameters have been measured for frequencies up to $1 \mathrm{GHz}$ and $250 \mathrm{MHz}$ for NGMM and NGMSM, respectively. DL data rate of each configuration is examined by applying conventional block diagonalization.
\end{abstract}

Index Terms-Multi-Pair Cable Measurement, C-RAN, Radio over Cable, 5G indoor service provisioning.

\section{INTRODUCTION}

With the rapid growth of various wired and wireless broadband applications such as smart homes and AR/VR, over $70 \%$ of mobile traffics is reported to occur indoors, and this percentage is expected to keep on increasing in the coming years. Taking fiber all the way to the access point is an attractive solution for high speed fixed and mobile access, however, it requires fiber infrastructure along each access path. The fiber optics deployment between the distribution and access point is the most expensive part of the access section, which may require digging along each path. This motivates the academia and industry to develop costeffective access solutions based on fiber to the distribution point (FTTdp) [1], [2], where the fiber optics is deployed to the distribution point only, while using the existing copper infrastructure (Ethernet, telephone) as the last mile from $20 \mathrm{~m}$ to $200 \mathrm{~m}$. For next-generation broadband access technology,
International Telecommunication Union - Telecommunication Standardization Sector (ITU-T) defines the G.fast standards G.9700 [3] and G.9701 [4] in 2014 that aims to achieve 1 Gbps using existing short loop (up to $100 \mathrm{~m}$ ) copper cables infrastructure. Radio over Copper (RoC) based wireless access architectures are proposed in [5], [6], [7], where the multi-pair copper cables are used in backhaul and fronthaul to achieve low cost and high data rate solution for cloud radio access network (C-RAN).

Telecommunication operators in coordination with vendors are exploring new directions to increase the bit rate of existing access technologies. Fifth generation $(5 \mathrm{G})$ cellular communication systems envision to fulfill the increasing demands of mobile broadband services by the indoor deployment of lowpower small base stations (BSs) [8]. C-RAN [9] proposed a centralized BSs architecture where the baseband units (BBUs) of small BSs are located at the centralized BBU pool, while the remote radio units (RRUs) are geographically distributed over a wide area to provide physical radio functionalities. The communication links between the BBU pool and each distributed RRUs are referred to as front-hauls. Various fronthaul architectures have been proposed for indoor coverage, however, their high design cost made then unfeasible for practical implementation. To resolve design complexities in front-haul solutions at extremely low cost, [7] introduces a novel distributed antenna access system for $5 \mathrm{G}$ indoor service provisioning where the Distributed Antenna Units (DAUs) are placed a few hundred meters from the RRU. The proposed design take the advantage of existing in-building LAN cable infrastructure to transport the analog signals between the DAUs and RRU. The primary objective of deploying LAN cable infrastructure in most of the commercial and residential buildings is to provide connectivity for telephone ( 2 pairs) and Ethernet (4 pairs) services, however, the use of wireless access technology i.e., wireless local area network (WLAN) results in unused copper wire infrastructure with in a building which is perfect for the deployment of next-generation access technology.

In addition to using multi-pair copper cables in the nextgeneration access network, the capacity of the system can further increase using Phantom mode signaling where the additional virtual twisted pairs are achieved by applying differential signal across two twisted pairs. The achievable 
data rate of multi-pair copper cables is the function of both cable length and transmission bandwidth [6]. Hence high data rate in copper-based wireless access solution can only be achieved by extending transmission bandwidth with shorter loop lengths, which constitutes extreme challenges in modeling and the measurements of a multi-pair copper channel as a transmission medium. The multi-pair copper channel is characterized by insertion loss (IL) over each twisted pair (TP) and the crosstalk among different TPs, which is due to the capacitive and inductive coupling between the neighboring TPs in a cable binder. Significant efforts have been made by the scientific community to model or measure the direct and crosstalk channels in a multi-pair copper channel. The classical empirical RLGC channel model is usually used to characterize the twisted pair copper cables, where the transmission medium describes as a cascade of identical cells with infinitesimal length [10], [11]. For the simulation purpose, the empirical multi-pair channel models based on American and European test loop configurations were developed and standardized [10], [11], [12]. The general consensus exists for the adoption of an empirical model of the direct channel, however, the empirical models for crosstalk channels are not accurate to predict the measured crosstalk channels [13], [14], [15]. Further, this channel model was only valid for frequencies up to $30 \mathrm{MHz}$ [11], [12]. D. Acatauassu et al. present the stochastic channel model for both time and frequency domains in [16]. The closed-form expressions with the low computational cost are presented for multi-pairs cable measurements up to $200 \mathrm{MHz}$.

Multi-pair copper channel characterization plays a very important role to evaluate the performance of the access system. Therefore, multi-pair cable measurement for direct and crosstalk channels is unavoidable for copper-based future access technologies. The method for refining the parameters of $1 \%$ worst-case crosstalk model is presented in [17], where CAT-5 cable is characterized for $212 \mathrm{MHz}$ channel bandwidth. Cable measurements for Phantom mode (PM) transmission over frequencies up to $300 \mathrm{MHz}$ are presented in [18], where the $50 \mathrm{~m}$ CAT-5e cable is characterized for IL and crosstalk. Worst case crosstalk measurements are presented in [19], where the authors proposed a measurement setup that does not require baluns, that are required to excite the balanced modes on Ethernet cables. The proposed measurement setup is not only complicated but also impractical for commercial use since it requires multiple vector network analyzers (VNA), a special fixture that replaces balun, non-conventional calibration techniques, and post-processing computations.

The focus of this paper is to design and evaluate the performance of the multi-pair cable system, where the deployment scenario is based on MIMO structure using multi-pair copper cable referred to as next-generation multi-pair MIMO (NGMM) and super MIMO (NGMSM).

\section{A. Next Generation Multi-pair MIMO (NGMM)}

In NGMM, multi-pair cable (2/4 pairs) uses the MIMO structure for single DAU. For multiple DAUs, the system is suffered from inter (among multiple cables) and intra (within single cable) cable crosstalk that makes complex crosstalk

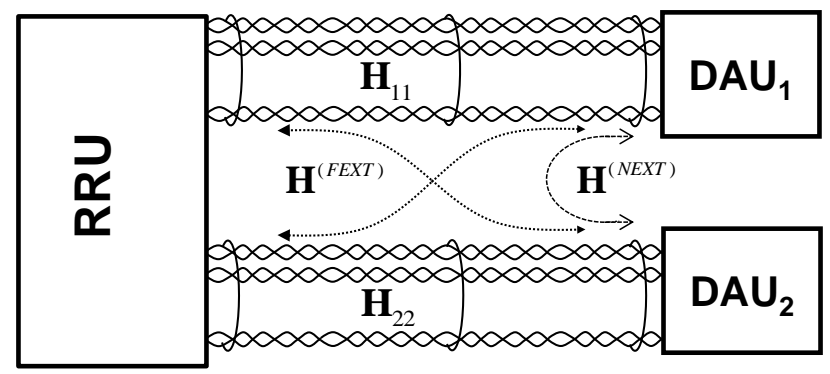

Fig. 1. Next-Generation Multi-pair MIMO (NGMM)

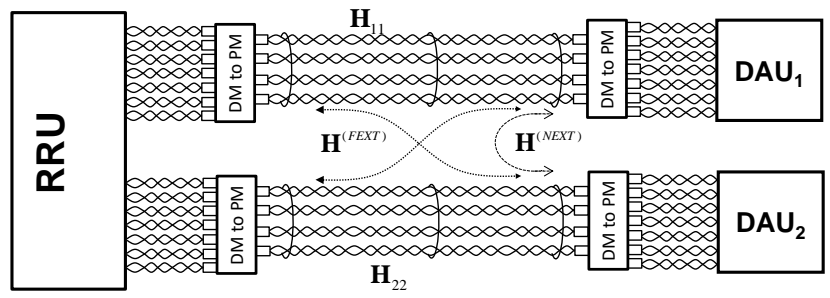

Fig. 2. Next Generation Multi-pair Super MIMO (NGMSM).

scenario within the system. The crosstalk scenario is shown in Fig. 1 for 2 DAUs that are connected to the RRU via multi-pair cables.

\section{B. Next-Generation Multi-pair Super MIMO (NGMSM)}

NGMSM uses a transformer-based circuit at RRU and DAU to create additional virtual pairs using an existing multipair cable. This technique is called Phantom mode and it is established using common-mode signals of physical cable pairs. The total number of pairs using phantom mode is $2 N-1$, where $N$ is the number of physical copper pairs in a multi-pair cable. Fig. 2 shows the NGMSM structure for Cat 5 cable, where the DM to PM unit represents the transformer-based circuit to create Phantom mode (PM) from the differential mode (DM) pairs.

The paper is organized as: the details of measurement devices and measurement setup for direct and crosstalk cable characteristics are given in Section II. The measurement scenario for NGMM and NGMSM channels are detailed in Section III. Measurement results including inter and intra cable crosstalk for DM and PM cable pairs are described in Section IV. The model for inter-pair interference mitigation in multicable environment is explained in $\mathrm{V}$. Conventional block diagonalization [20], [21] based on singular value decomposition (SVD) for interference cancellation in the downlink (DL) is in Section VI, while the numerical results for achievable DL capacity using the measured NGMM and NGMSM channels are presented in Section VII. At last, the paper is concluded in Section VIII.

\section{Cables Channel Measurement Setup for NGMM AND NGMSM}

The achievable bi-directional data rate depends mainly on copper wire channel characteristics in the CAT cables binder. The accurate channel characteristics are required to better 
estimate the achievable data rate using short loop length copper cables in NGMM and NGMSM systems. The following devices and equipment were used to properly setup the measurement environment:

PNA-X Network Analyzer Network analyzer measure Sparameters (i.e $S_{11}, S_{21}, S_{12}, S_{22}$ ) to characterize two-port networks such as amplifiers and filters. It contains both a source and multiple receivers and generally extracts relative amplitude and phase information (frequency or power sweeps). In our measurement setup, we have used Agilent 2/4 port PNA-X network analyzer N5242A with the frequency range from $10 \mathrm{MHz}$ to $26.5 \mathrm{GHz}, 4$ ports with two built-in sources, Output power of $+18 \mathrm{dBm}$.

Calibration Kit characterizing systematic error and removing its effect from subsequent measurements is accomplished through Vector-error correction. Two-port calibration requires measurements on four standards short-openload-through or SOLT. The standards are defined in a calkit definition file, which is stored in the Agilent PNA. To make accurate measurements, we ensure that the cal-kit definition matches the actual calibration kit used for the measurement. In our measurement setup, we have used 2 port calibration kit NA-4691 60008 with the frequency range from $300 \mathrm{kHz}$ to $26.5 \mathrm{GHz}$.

Balun Transformer Balun converts a balanced signal (two signals working against each other where the ground is irrelevant) to an unbalanced signal (a single signal working against ground or pseudo-ground). High frequency Balun transformer NH16447 is used in the measurement setup to transform signals between the coaxial cable and twisted pair. It provides an easy and straightforward means of measuring circuit characteristics to $1.2 \mathrm{GHz}$.

In order to completely characterize an unknown linear twoport device, measurements under various conditions are conducted and a set of parameters are computed. These parameters completely describe the electrical behavior of the device (or network) under test. S-parameters are used to characterize high-frequency networks by means of familiar measurements such as gain, loss, and reflection coefficient. The measured $\mathrm{S}$-parameters of multiple devices can be cascaded to predict overall system performance. S-parameters are easily imported and used for circuit simulations in electronic-design automation (EDA) tools like Agilentâs Advanced Design System (ADS) and MATLAB. Here, we now explain the measurement setup for the multi-pair cable parameters, i.e., characteristics impedance, insertion loss (IL), Far End Crosstalk (FEXT), and Near End Crosstalk (NEXT).

\section{A. Characteristics Impedance}

Transmission lines (i.e. twisted pairs/ coaxial cables) are used to convey RF energy from one point to another with minimal loss. Each transmission line has a characteristic impedance $\left(Z_{0}\right)$ and termination at the end of a transmission line must match the characteristic impedance of the line to prevent loss of energy due to reflections. When the transmission line is terminated in its characteristic impedance, maximum power is transferred to the load. When the termination is not $Z_{0}$, the

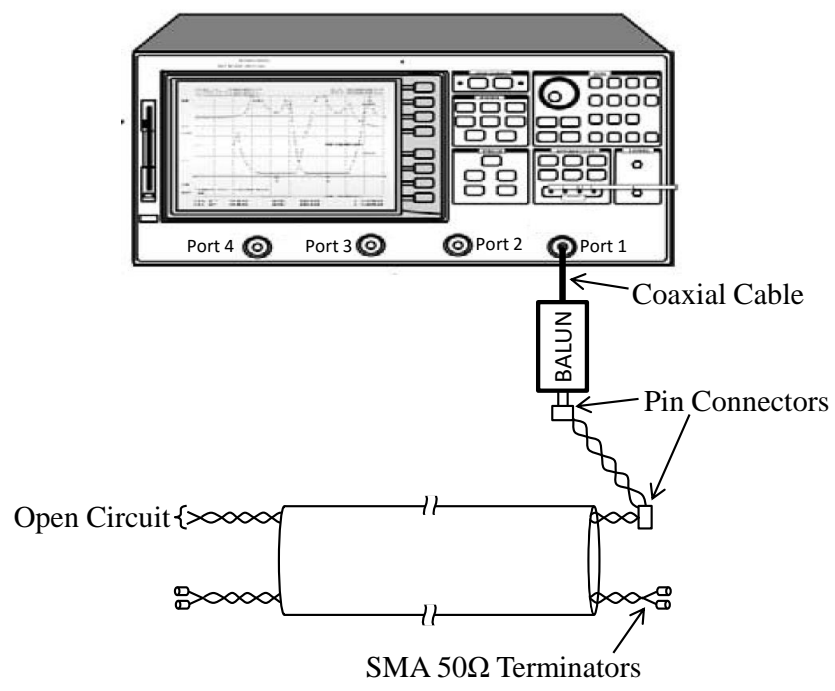

a. Open Circuit Configuration

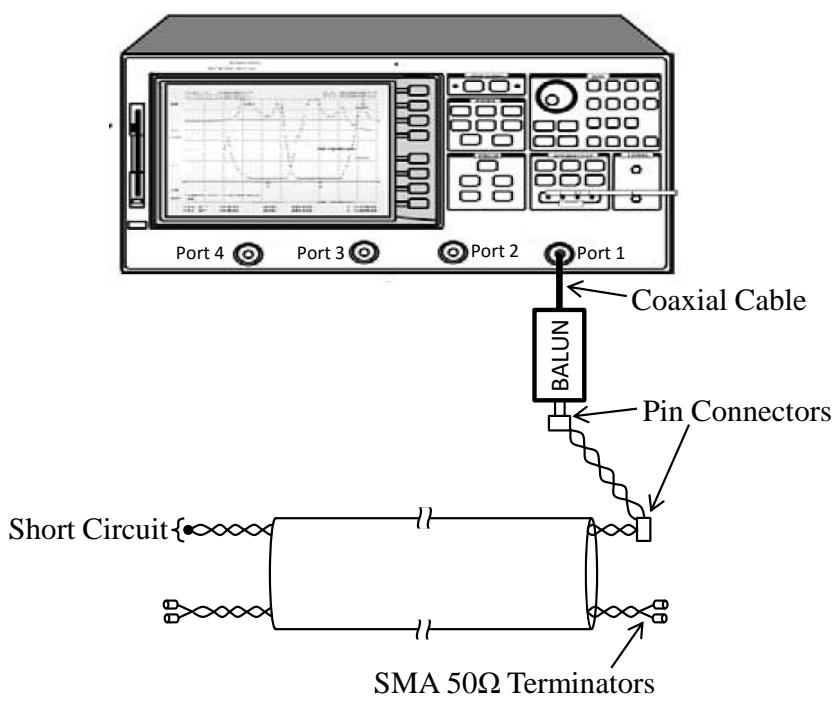

b. Short Circuit Configuration

Fig. 3. Characteristics Impedance Measurement Setup.

portion of the signal which is not absorbed by the load is reflected back toward the source. Characteristics Impedance is measured through open-circuit impedance $\left(Z_{o c}\right)$ (with the other end of the line open; that is, not connected to anything) and short-circuit impedance $Z_{s c}$ (with the other end of the line short-circuited) by $Z_{0}=\sqrt{Z_{o c} Z_{s c}}$. The measurement setup is shown in Fig. 3 for open-circuit (a) and short-circuit (b), where S-parameter $S_{11}$ is measure on PNA to compute open opencircuit impedance $\left(Z_{O c}\right)$ and short-circuit impedance $\left(Z_{S c}\right)$ for $Z_{\text {ref }}=100 \Omega$ by

$$
\begin{aligned}
& Z_{o c}=Z_{\text {ref }}\left(\frac{1+S_{11}}{1-S_{11}}\right) \\
& Z_{s c}=Z_{\text {ref }}\left(\frac{1+S_{11}}{1-S_{11}}\right) .
\end{aligned}
$$




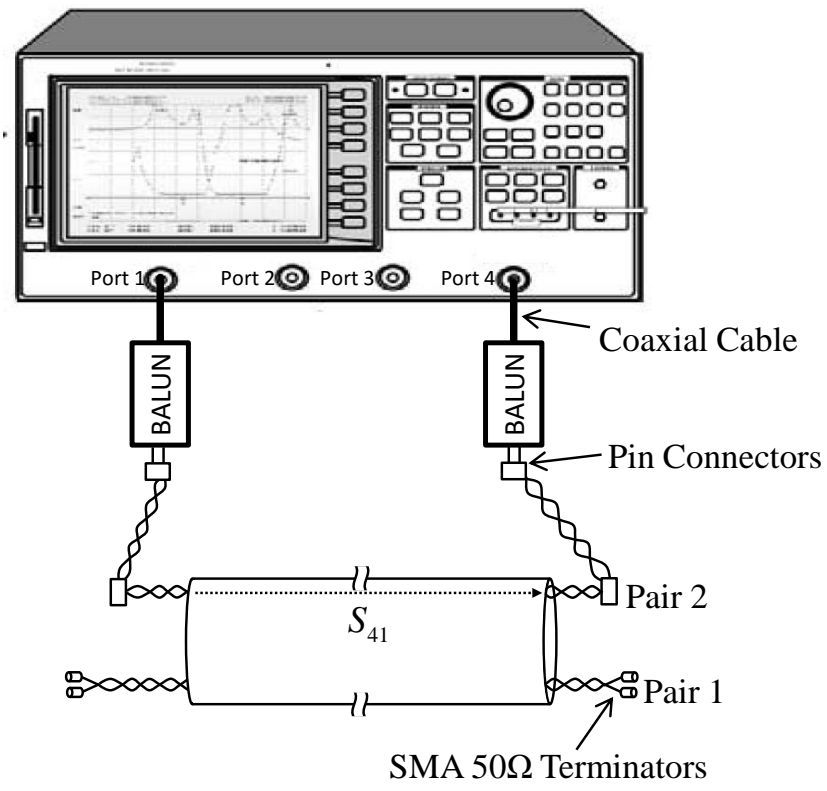

Fig. 4. IL Measurement Setup.

\section{B. Insertion Loss (IL)}

IL is measured between the opposite ends of a single twisted pair in a cable binder contains multiple twisted pairs. The measurement setup for IL is shown in Fig. 4, where one end of twisted pair 1 is connected to port 1 and the opposite end is connected to port 4 . All the ends of the remaining twisted pairs are properly terminated using $50 \Omega$ SMA resistors. The IL between two ends of a twisted pair is measured by S-parameter $S_{41}$ on PNA.

\section{Far-End Crosstalk (FEXT)}

FEXT is defined as the crosstalk effect between a pair of transceivers located at opposite ends of two separate pairs within the same cable binder. In other words, the FEXT noise at a particular transceiver is caused by signals transmitted by transceivers of other pairs at the opposite end of the twisted cable. The measurement setup for FEXT is shown in Fig. 5, where twisted pair 1 is connected to port 1 and twisted pair 2 is connected to port 4 . The FEXT between pair 1 and pair 2 is measured by S-parameter $S_{41}$ on PNA where the other ends of twisted pairs are properly terminated using $50 \Omega$ SMA resistors.

\section{Near-End Crosstalk (FEXT)}

NEXT is defined as the crosstalk effect between a pair of transceivers that transmit and receive signals at the same end of twisted pair cables that share the same frequency band. In other words, the NEXT noise at a particular transceiver is caused by signals transmitted by other transceivers at the same end of the twisted cable. The measurement setup for NEXT is shown in Fig. 6, where twisted pair 1 is connected to port 1 and twisted pair 2 is connected to port 4 . The NEXT between pair 1 and pair 2 is measured by S-parameter $S_{41}$ on PNA where the other ends of twisted pairs are properly terminated using $50 \Omega$ SMA resistors.

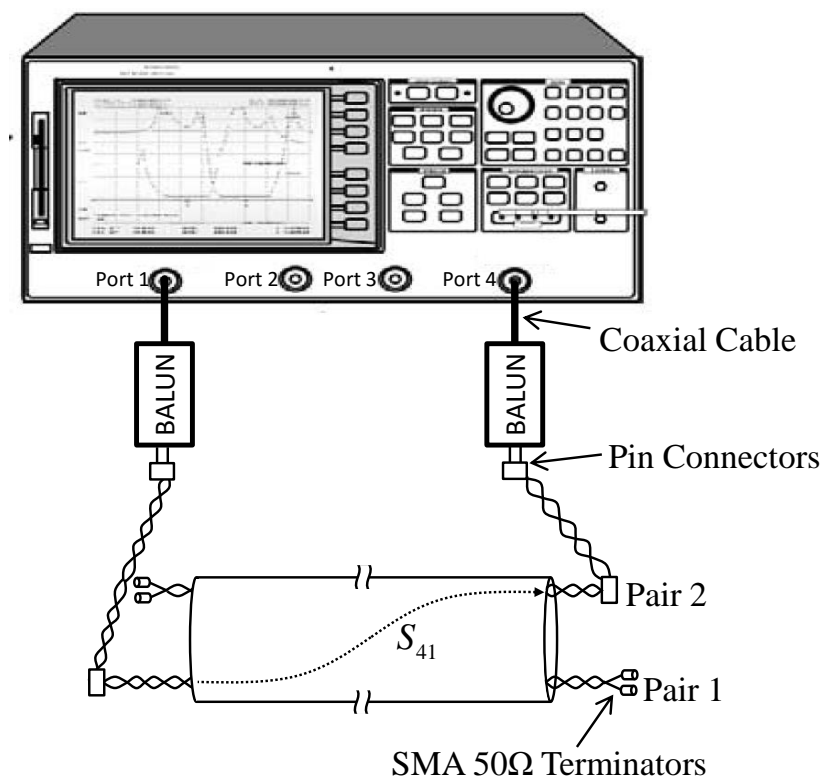

Fig. 5. FEXT Measurement Setup.

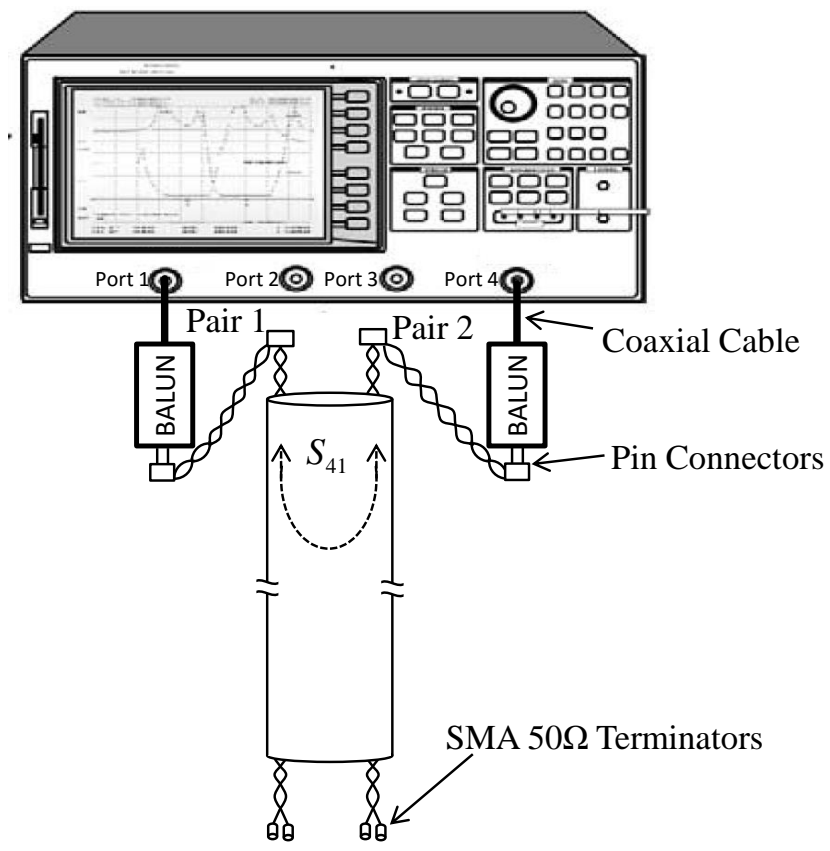

Fig. 6. NEXT Measurement Setup.

\section{NGMM AND NGMSM MEASUREMENT SETUP}

CAT-5 cables are commonly used to establish indoor networking, therefore we use it to create the measurement setup for NGMM and NGMSM scenarios. In the building, many CAT-5 pairs tied together to provide Ethernet services to different offices/homes at the different locations as shown in Fig. 7. The cable standard provides the performance of the CAT 5 cables up to $100 \mathrm{MHz}$ that is suitable for 10BASE-T, 100BASE-TX (Fast Ethernet), and 1000BASE-T (Gigabit Ethernet), however the cable characteristics for intended NGMM and NGMSM frequency band (i.e., $1 \mathrm{GHz}$ ) is not publicly 


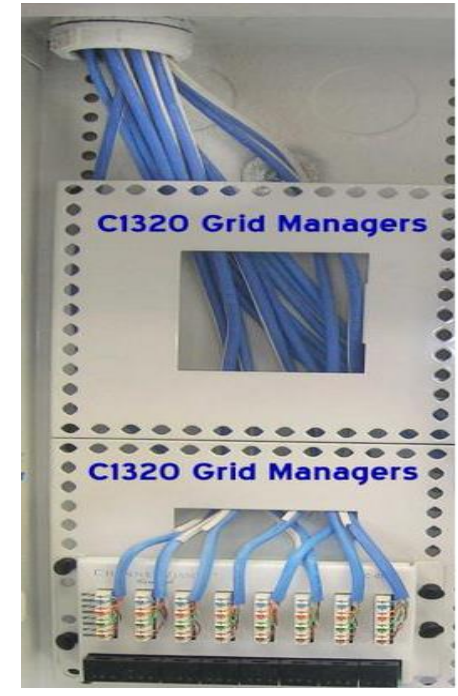

Fig. 7. Multiple CAT-5 cables from distribution point.

available.

To make the measurement for a practical scenario, we tied 2 CAT-5 cables together to measure inter cable crosstalk parameters with the same loop length. Pin connectors are used to establish connectivity between network analyzer and twisted pair because it is already proven that the performance of using pin connectors and RJ-45 connectors are the same, however, pin connectors enable to use the same cable for NGMM and NGMSM testing. Measurement parameters for NGMM and NGMSM scenarios are as follows:

1) Frequency Range:

NGMM: $10 \mathrm{MHz}-1 \mathrm{GHz}$

NGMSM: $10 \mathrm{MHz}-300 \mathrm{MHz}$ (Note: the frequency response of the transformers used to establish phantom mode (PM) is limited to $250 \mathrm{MHz}$ )

2) Number of Twisted Pairs:

NGMM: 8 pairs with 4 pairs per CAT-5 cable

NGMSM: 14 pairs with 4 physical pairs and 3 virtual pairs per CAT-5 cable.

3) Connector Type: Pin

4) Measurement Parameters for NGMM:

Characteristics-impedance of the twisted pair.

IL Insertion Loss: Direct channel response for the frequency range.

FEXT Far-End Crosstalk: Cross-coupling of the signals on other pairs at the receiver end

a) Intra FEXT (FEXT coupling between the twisted pairs of same CAT-5 cable)

b) Inter FEXT (FEXT coupling between the twisted pairs of different CAT-5 cables)

NEXT Near-End Crosstalk: Cross-coupling of the signals on other lines at the transmitter end

a) Intra NEXT (reverse crosstalk coupling between the twisted pairs of same CAT-5 cable)

b) Inter NEXT (reverse crosstalk coupling between the twisted pairs of different CAT-5 cables)
5) Measurement Parameters for NGMSM:

Characteristics-impedance of the twisted pair.

IL Insertion Loss: Direct channel response for the frequency range.

a) Differential Mode (DM)

b) Phantom Mode (PM)

FEXT Far-End Crosstalk: Cross-coupling of the signals on other lines at the receiver end

a) Intra FEXT (forward crosstalk coupling between the twisted pairs of same CAT-5 cable). FEXT are measured between the pairs with in CAT- 5 cable 1 or 2 as

i) DM (1/2) to DM (1/2): FEXT coupling between the DM pairs.

ii) DM (1/2) to PM (1/2): FEXT coupling from the DM pairs to the PM pairs.

iii) PM (1/2) to DM (1/2): FEXT coupling from the PM pairs to the DM pairs.

iv) PM (1/2) to PM (1/2): FEXT coupling between the PM pairs

b) Inter FEXT (forward crosstalk coupling between the twisted pairs of different CAT-5 cables). FEXT is measured between the pairs of two CAT-5 cable as

i) DM (1/2) to DM (2/1): cross-coupling between the DM pairs of Cable 1 to DM pairs of Cable 2 , and vice versa.

ii) DM (1/2) to PM (2/1): cross-coupling between the DM pairs of Cable 1 to PM pairs of Cable 2 , and vice versa.

iii) PM (1/2) to DM (2/1): cross-coupling between the PM pairs of Cable 1 to DM pairs of Cable 2 , and vice versa.

iv) PM (1/2) to PM (2/1): cross-coupling between the PM pairs of Cable 1 to PM pairs of Cable 2 , and vice versa.

NEXT Near End Crosstalk: Coupling of the direct signals on other lines at the transmitter end.

a) Intra NEXT (reverse crosstalk coupling between the twisted pairs of same CAT-5 cable)

i) DM (1/2) to DM (1/2): NEXT coupling between the DM pairs.

ii) DM (1/2) to PM (1/2): NEXT coupling from the DM pairs to the PM pairs.

iii) PM (1/2) to DM (1/2): NEXT coupling from the PM pairs to the DM pairs.

iv) PM (1/2) to PM (1/2): NEXT coupling between the PM pairs

b) Inter NEXT (reverse crosstalk coupling between the twisted pairs of different CAT-5 cables)

i) $\mathrm{DM}(1 / 2)$ to $\mathrm{DM}(2 / 1)$ : NEXT between the DM pairs of Cable 1 to DM pairs of Cable 2, and vice versa.

ii) DM (1/2) to PM (2/1): NEXT between the DM pairs of Cable 1 to PM pairs of Cable 2, and vice versa. 
iii) PM (1/2) to DM (2/1): NEXT between the PM pairs of Cable 1 to DM pairs of Cable 2, and vice versa.

iv) PM (1/2) to PM (2/1): NEXT between the PM pairs of Cable 1 to PM pairs of Cable 2, and vice versa.

\section{A. Phantom Mode (PM) Circuit}

DSL Phantom Mode technology is a bit of magic for the copper network. It creates a third (phantom or virtual) channel over 2 regular copper pairs. Phantom mode transmission over 2 pairs exploits the difference between the common mode voltages of the 2 pairs. To preserve the balance and avoid excess egress and ingress noise, typically hierarchical phantoms (or super-phantoms) are used in which case the number of pairs $(N)$ is a power of $2\left(N=2^{n}\right)$. The naming is introduced to make a distinction with the definition of common-mode transmission, where the common ground of 1 twisted pair is used as a reference. In general, when $N$ wire pairs are available, $N$ normal (differential) channels and $N-1$ phantom channels can be used.

Fig. 8 shows the structure of Phantom mode for CAT 5 cable, where broadband center tap transformers are used to create virtual pairs (Phantom Mode). We have used highfrequency broadband transformers that are specially designed for this measurement scenario. The frequency range of operation of these transformers is up to $220 \mathrm{MHz}$. There are four differential mode pairs and 3 Phantom mode pairs achieved through this configuration.

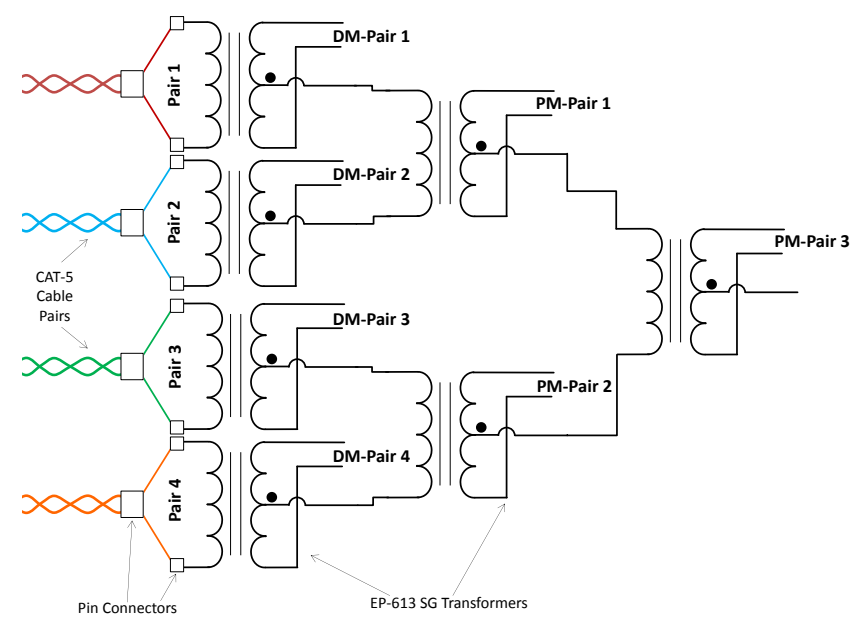

Fig. 8. Phantom Mode for CAT-5 cable using high frequency transformers.

\section{Measurement Results}

The measurement for all the given scenario has been performed for the following settings at PNA:

1) Stop Frequency: $1 \mathrm{GHz}$ (NGMM)/ $220 \mathrm{MHz}$ (NGMSM)

2) Number of Points $=10000$

3) Average IF: $100 \mathrm{kHz}$

4) Traces $\left(S_{11}, S_{14}, S_{41}, S_{44}\right)$

The measurement results were saved in ".csv" format and then use in Matlab for further processing.
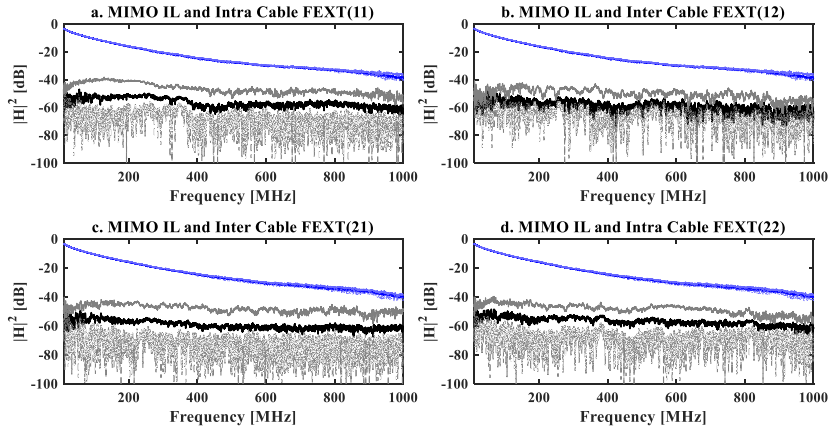

$\operatorname{mean}_{i}\left|h_{i i}\right|^{2}-\cdots \cdot \min _{i}\left|h_{i i}\right|^{2}-\max _{i}\left|h_{i i}\right|^{2}-\operatorname{mean}_{i}\left|h_{i j}\right|^{2}-\cdots \min _{i}\left|h_{i j}\right|^{2}-\max _{i}\left|h_{i j}\right|^{2}$

Fig. 9. IL Vs FEXT CAT-5 Cable MIMO Measurement.
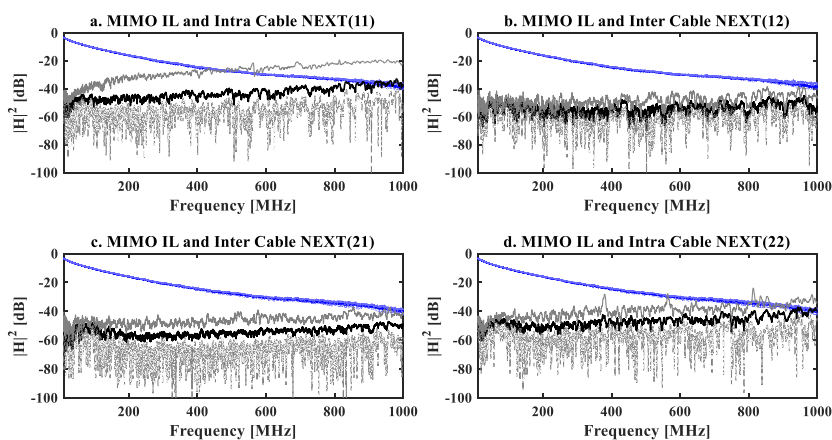

$\operatorname{mean}_{i}\left|h_{i j}\right|^{2}-\ldots \min _{i}\left|h_{i j}\right|^{2}-\max _{i}\left|h_{i j}\right|^{2}-\operatorname{mean}_{i}\left|h_{i j}\right|^{2}-\ldots \cdot \min _{i}\left|h_{i j}\right|^{2}-\max _{i}\left|h_{i j}\right|^{2}$

Fig. 10. IL Vs NEXT CAT-5 Cable MIMO Measurement.

\section{A. NGMM Channel}

2-50m-CAT-5-Cable-MIMO: The measurement results of two tied 50m CAT-5 cables (4 twisted pairs/cable) are present in this section. The couplings within the cable and in between the cables are termed as Intra and Inter crosstalk coupling respectively. The convention used to represent inter and intra coupling in the measurement results is Coupling ( $j i)$, where Coupling is either FEXT or NEXT, " $i$ " shows the disturber cable, and " $j$ " represent the victim cable. As an example, the NEXT(12) shows the inter cable near end coupling from cable 2 to cable 1 , here the disturber cable is 2 , and the victim cable is 1 .

\section{IL Vs FEXT}

Measurement results for IL vs FEXT for NGMM are shown in Fig. 9, where IL is reduced to $-40 \mathrm{~dB}$ at $1 \mathrm{GHz}$. The FEXT is approximately the same for inter and intra cables environment.

\section{IL Vs NEXT}

IL and NEXT coupling for NGMM is shown in Fig. 10, where the NEXT coupling is quite strong as compared to the FEXT. The strength of intra cable NEXT is much stronger than the inter cable NEXT, as expected, due to the shield covering on each CAT-5 cable. In cable 1, 90\% worst case NEXT coupling is stronger than IL at $600 \mathrm{MHz}$, however, this effect can be observed at $900 \mathrm{MHz}$ in cable 2 . The FEXT is approximately the same for inter and intra cables environment. 


\section{B. NGMSM Channel}

The measurement results of the Phantom mode of two tied 50m CAT-5 cables (4 twisted pairs/cable) or 2 parallel Super MIMO channels are present in this section. All the coupling scenario mentioned in Section III for Intra and Inter crosstalk are discussed in this section. The convention used to represent coupling in the measurement results is through Coupling $(j i)$ mode(i) to mode(j), where mode(i) to mode(j) represent the coupling between different modes of Super MIMO, Coupling is either FEXT or NEXT, " $i$ " shows the disturber cable and $" j "$ represent the victim cable. As an example the NEXT(21)$\mathrm{DM}(1)$ to $\mathrm{PM}(2)$ shows the inter cable NEXT coupling from Differential Mode pairs of cable 1 to Phantom Mode pairs of cable 2 , here the disturber cable is 1 , and the victim cable is 2 .

\section{1) IL-Vs-FEXT:}

\section{1) Intra FEXT Coupling}

The measurement results for intra cable IL Vs FEXT at cable 1 and 2 are shown in Fig. 11 and Fig. 12 respectively. The $90 \%$ worst case intra cable FEXT coupling among DM pairs is about $-45 \mathrm{~dB}$ while IL of DM pairs are reduced to $-20 \mathrm{~dB}$ at $220 \mathrm{MHz}$. FEXT coupling between $\mathrm{DM}$ and PM pairs (and vice versa) is increased to $-40 \mathrm{~dB}$, however, some deep notches have been observed in IL of PM pairs 5 and 6 for cable 1 and 2 at frequency $150 \mathrm{MHz}$ while IL of PM pair 7 is relatively better than pairs 5 and 6 . The deep notches may be the result of the transformer mismatch because the characteristic impedance of the cable with PM circuit might change and that we did not consider in the measurements. The IL of PM pairs is reduced to $-25 \mathrm{~dB}$ at $220 \mathrm{MHz}$. The FEXT among PM pairs in cable 1 and 2 is very strong and its power is much higher than IL of PM i.e. at frequency $150 \mathrm{MHz}$.

\section{2) Inter FEXT Coupling}

Inter cable IL Vs FEXT measurement results at cable 1 from cable 2 and at cable 2 from cable 1 are shown in Fig.13 and Fig.14 respectively. The 90\% worst-case FEXT coupling among DM pairs of two cables is reduced to $-48 \mathrm{~dB}$, however, the FEXT coupling between PM pairs of cable 2 and DM pairs of cable 1 (and vice versa) is reduced to around $-50 \mathrm{~dB}$. The inter FEXT coupling between PM pairs of cable 1 and 2 (and vice versa) is much weaker than intra FEXT coupling and it reaches $-30 \mathrm{~dB}$ in $90 \%$ worst-case scenario.

\section{2) $I L-V s-N E X T$ :}

\section{1) Intra NEXT Coupling}

Intra cable IL Vs NEXT at cable 1 and 2 are measured and results are shown in Fig. 15 and Fig. 16 respectively. The $90 \%$ worst intra cable NEXT among the DM pairs is $-40 \mathrm{~dB}$ as can be observed in the plots. The increase in NEXT coupling to $-30 \mathrm{~dB}$ can also be observed between DM and PM pairs (and vice versa), and it is quite close to $\mathrm{IL}$ at around $220 \mathrm{MHz}$. The NEXT coupling among PM pairs in cable 1 and 2 is very strong and its dominate IL of PM for frequencies greater than $100 \mathrm{MHz}$.
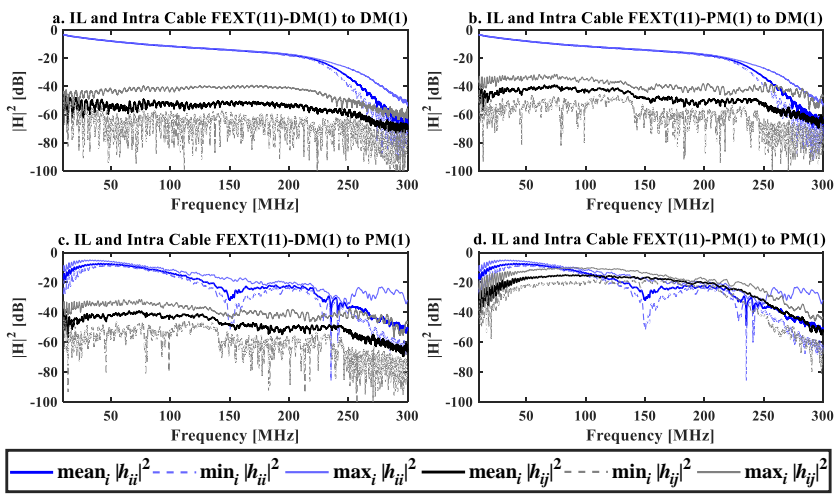

Fig. 11. IL and Intra FEXT Coupling at CAT-5 Cable 1.
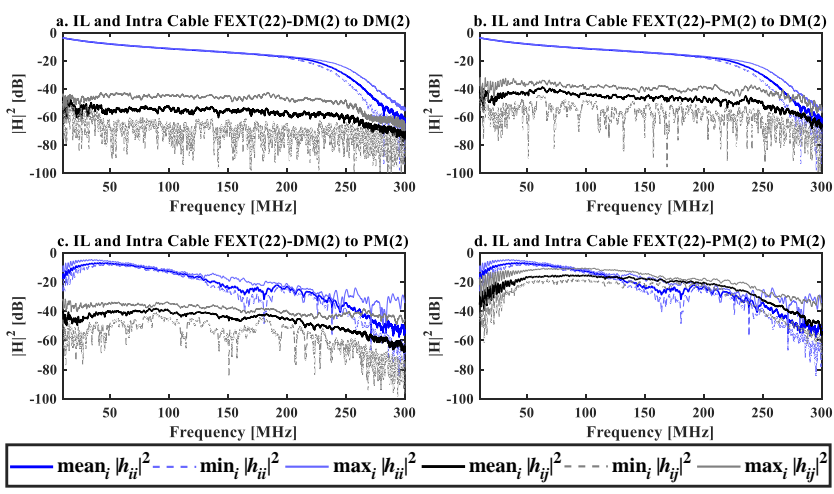

Fig. 12. Intra FEXT Coupling at CAT-5 Cable 2.
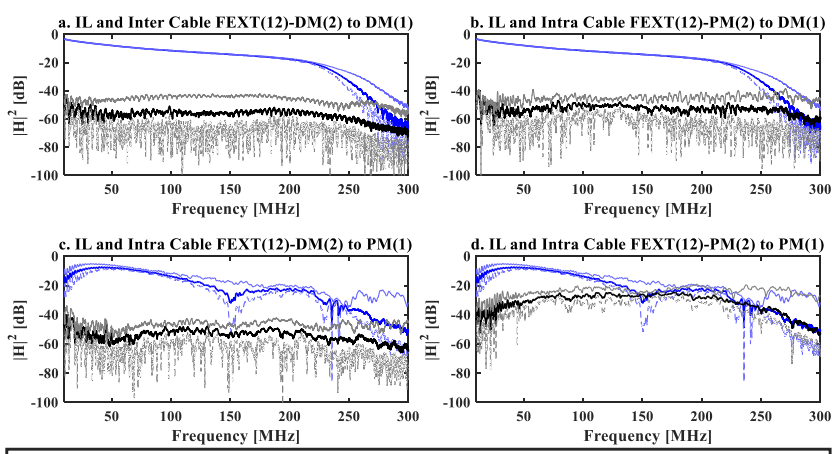

$\operatorname{mean}_{i}\left|h_{i i}\right|^{2}-\cdots \cdot \min _{i}\left|h_{i i}\right|^{2}-\max _{i}\left|h_{i i}\right|^{2}-\operatorname{mean}_{i}\left|h_{i j}\right|^{2}-\cdots \cdot \min _{i}\left|h_{i j}\right|^{2}-\max _{i}\left|h_{i j}\right|^{2}$

Fig. 13. Inter FEXT Coupling at CAT-5 Cable 1.
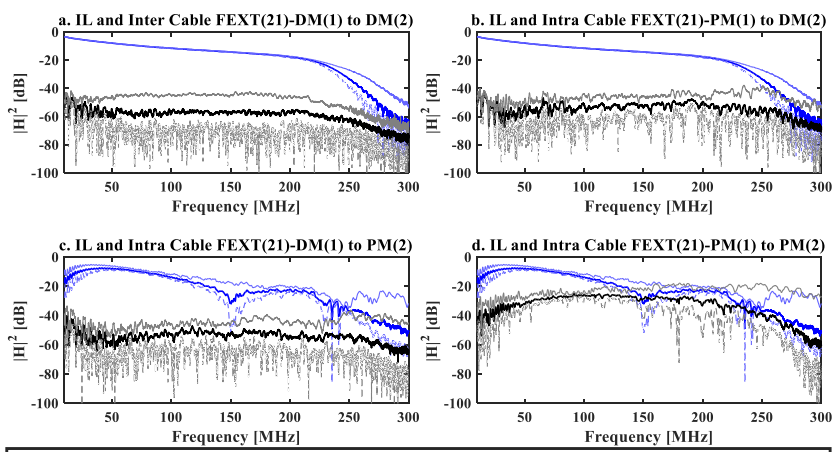

$\operatorname{mean}_{i}\left|h_{i i}\right|^{2}-\ldots \cdot \min _{i}\left|h_{i i}\right|^{2}-\max _{i}\left|h_{i i}\right|^{2}-\operatorname{mean}_{i}\left|h_{i j}\right|^{2}-\cdots \cdot \min _{i}\left|h_{i j}\right|^{2}-\max _{i}\left|h_{i j}\right|^{2}$

Fig. 14. Inter FEXT Coupling at CAT-5 Cable 2. 

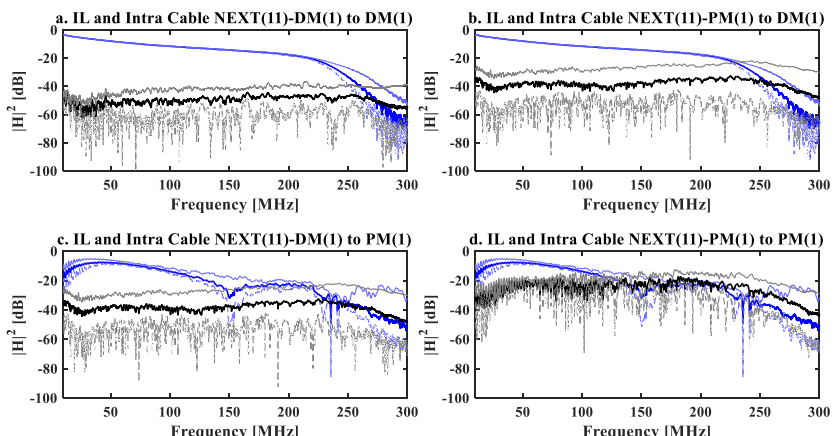

$\operatorname{mean}_{i}\left|h_{i i}\right|^{2}-\cdots \cdot \min _{i}\left|h_{i i}\right|^{2}-\max _{i}\left|h_{i i}\right|^{2}-\operatorname{mean}_{i}\left|h_{i j}\right|^{2}-\cdots \min _{i}\left|h_{i j}\right|^{2}-\max _{i}\left|h_{i j}\right|^{2}$

Fig. 15. Intra NEXT Coupling at CAT-5 Cable 1.
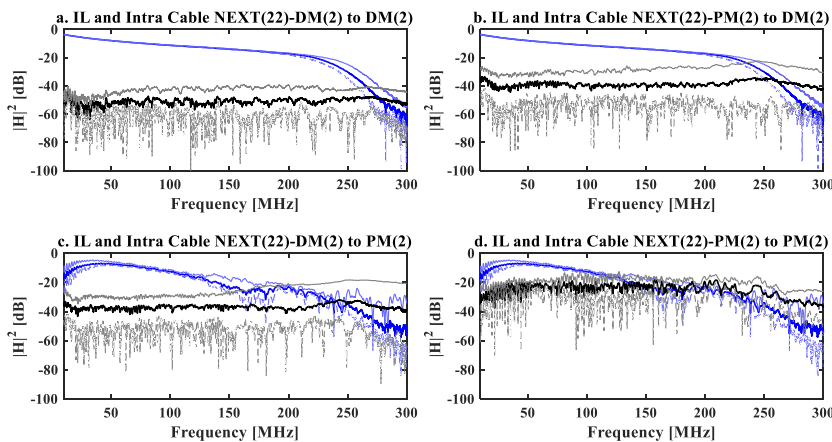

$\operatorname{mean}_{i}\left|h_{i i}\right|^{2}--\cdot \min _{i}\left|h_{i i}\right|^{2}-\max _{i}\left|h_{i i}\right|^{2}-\operatorname{mean}_{i}\left|h_{i j}\right|^{2}-\cdots \cdot \min _{i}\left|h_{i j}\right|^{2}-\max _{i}\left|h_{i j}\right|^{2}$

Fig. 16. Intra NEXT Coupling at CAT-5 Cable 2.

\section{2) Inter NEXT Coupling}

Measurement results of inter cable NEXT at cable 1 from cable 2 and at cable 2 from cable 1 are shown in Fig. 17 and Fig. 18 respectively. The NEXT coupling among the DM pairs of two cables is relatively low as compared to the intra NEXT coupling and its strength is decreased to $-50 \mathrm{~dB}$. The maximum NEXT coupling between PM pairs of cable 2 and DM pairs of cable 1 (and vice versa) is also reduced to around $-40 \mathrm{~dB}$ at $250 \mathrm{MHz}$ as compared to intra NEXT coupling. The level of NEXT coupling is also decreased between PM pairs of cable 1 and 2 (and vice versa) but it still approaches to IL of PM pairs at $150 \mathrm{MHz}$.
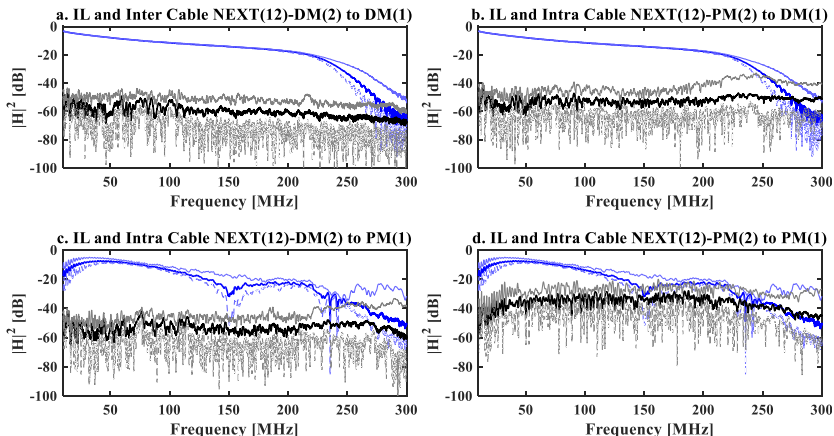

$-\operatorname{mean}_{i}\left|h_{i i}\right|^{2}-\cdots \min _{i}\left|h_{i i}\right|^{2}-\max _{i}\left|h_{i i}\right|^{2}-\operatorname{mean}_{i}\left|h_{i j}\right|^{2}-\cdots \cdot \min _{i}\left|h_{i j}\right|^{2}-\max _{i}\left|h_{i j}\right|^{2}$

Fig. 17. Inter NEXT Coupling at CAT-5 Cable 1.
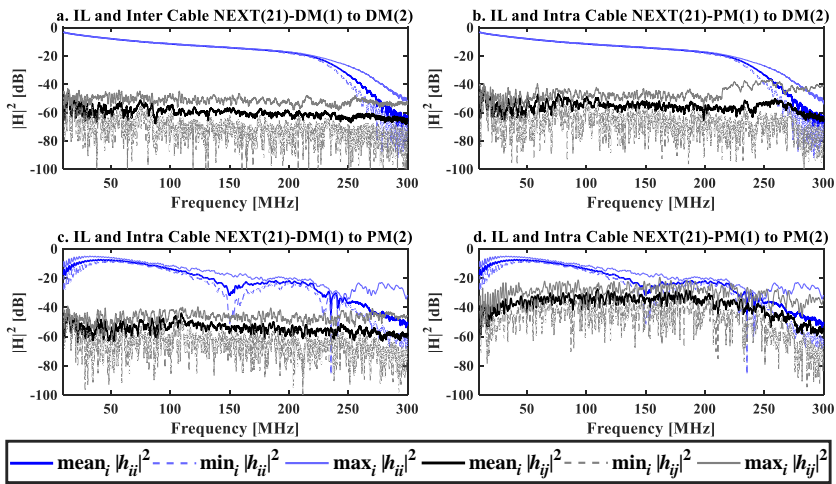

Fig. 18. Inter NEXT Coupling at CAT-5 Cable 2.

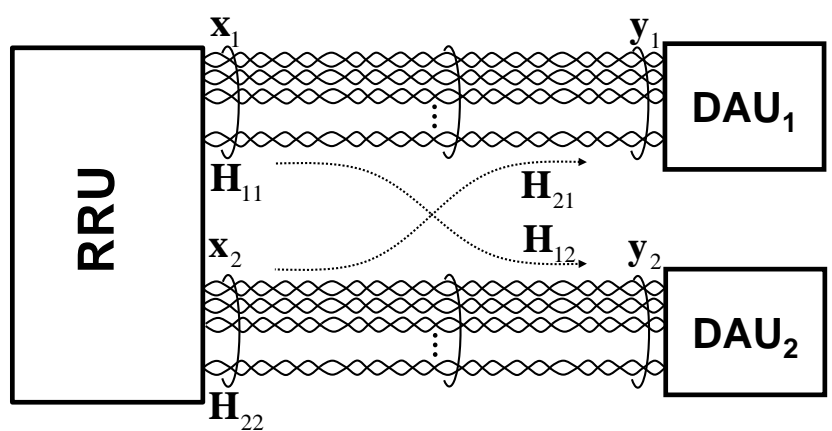

Fig. 19. NGMM system model for 2 DAUs in DL.

\section{NGMM SYSTEM MODEL}

To evaluate the capacity of NGMM, system model for DL is considered in Fig. 19, where each DAU uses MIMO technology to enhance the data rate. Fig. 19 shows 2 DAUs with $N$ twisted pairs each that are mutually interfering with one another. The ensemble of all $2 N \times 1$ received signals $\mathbf{y}(t)$ at time $\mathrm{t}$ is given by

$$
\begin{gathered}
\underbrace{\left[\begin{array}{l}
\mathbf{y}_{1}(t) \\
\mathbf{y}_{2}(t)
\end{array}\right]}_{\mathbf{y}(t)}=\underbrace{\left[\begin{array}{ll}
\mathbf{H}_{11} & \mathbf{H}_{12} \\
\mathbf{H}_{21} & \mathbf{H}_{22}
\end{array}\right]}_{\mathbf{H}} \underbrace{\left[\begin{array}{l}
\mathbf{x}_{1}(t) \\
\mathbf{x}_{2}(t)
\end{array}\right]}_{\mathbf{x}(t)}+\underbrace{\left[\begin{array}{l}
\mathbf{w}_{1}(t) \\
\mathbf{w}_{2}(t)
\end{array}\right]}_{\mathbf{w}(t)} \\
\mathbf{y}(t)=\underbrace{\left[\begin{array}{l}
\mathbf{H}_{1} \\
\mathbf{H}_{2}
\end{array}\right]}_{\mathbf{H}} \mathbf{x}(t)+\mathbf{w}(t),
\end{gathered}
$$

where $\mathbf{x}_{k}(t)=\left[x_{k, 1}(t), x_{k, 2}(t), \ldots, x_{k, N}(t)\right]^{T}$ denotes the signals from the finite alphabet $\Lambda$ of M-QAM constellation that are precoded and transmitted from $N$ twisted pairs belonging to the $k$ th DAU. The ensemble of $2 N \times 1$ transmitted signals related to all DAUs are in $\mathbf{x}(t)$. Each $N \times N$ matrix $\mathbf{H}_{i j}$ is the channel from $N$ lines of the $j$ th DAU towards the $i$ th DAU $(j \rightarrow i)$ and it accounts for the channel including the intracable FEXT (when $i=j$ ) and inter-cable-FEXT (when $i \neq j$ ). $\mathbf{H}$ is the $2 N \times 2 N$ channel matrix of the multi DAU system. The AWGN $\mathbf{w}_{k}(t)$ is uncorrelated among lines and DAUs, with the same power: $\mathbf{w}(t) \sim C N\left(0, \sigma^{2} \mathbf{I}\right)$. 


\section{DL BLOCK PRECODING FOR NGMM}

The general block diagram for DL block precoding is shown in Fig. 20, where inter and intra cable FEXT cancellation is achieved through linear block processing perform at the transmitter and receiver, as in [20], [21]. The $N \times 1$ ensemble signal $\tilde{\mathbf{y}}_{k}$ at the decision variable is

$$
\tilde{\mathbf{y}}_{k}=\mathbf{D}_{k}\left(\mathbf{H}_{k} \mathbf{F s}+\mathbf{w}_{k}\right),
$$

where $\mathbf{s}$ is a symbol vector of dimension $2 N \times 1$ for all the DAUs. The transmitted signal $\mathbf{x}$ is achieved through precoding filter $\mathbf{F}$ as

$$
\begin{aligned}
\mathbf{x} & =\mathbf{F s} \\
& =\mathbf{F}_{1} \mathbf{s}_{1}+\mathbf{F}_{2} \mathbf{s}_{2} .
\end{aligned}
$$

The ensemble of $N \times 1$ received signal $\mathbf{y}_{k}$ at $k$ th DAU is passed through the demodulation filter $\mathbf{D}_{k}$ to achieve $\tilde{\mathbf{y}}_{k}$, that is further processed by the maximum likelihood (ML) estimator to estimate the original transmitted symbol $\hat{\mathbf{s}}_{k}$. The channel matrix from the RRU to the $k$ th DAU is denoted by $\mathbf{H}_{k}$ and accordingly the precoding filter by $\mathbf{F}_{k}$. The signal received at $k$ th DAU is

$$
\begin{gathered}
\mathbf{y}_{k}=\mathbf{H}_{k} \mathbf{x}+\mathbf{w}_{k} \\
\mathbf{y}_{k}=\mathbf{H}_{k} \mathbf{F}_{k} \mathbf{s}_{k}+\mathbf{H}_{k} \tilde{\mathbf{F}}_{k} \tilde{\mathbf{s}}_{k}+\mathbf{w}_{k},
\end{gathered}
$$

where $\tilde{\mathbf{F}}_{k}$ and $\tilde{\mathbf{s}}_{k}$ are the modulation filter and the symbol vector, respectively, for the DAU other than $k$ th DAU. The matrix $\tilde{\mathbf{F}}_{k}$ and symbol vector $\tilde{\mathbf{s}}_{k}$ for $\mathrm{RRU}_{1}$ and $\mathrm{RRU}_{2}$ are defined as

$$
\begin{aligned}
\tilde{\mathbf{F}}_{1} & =\mathbf{F}_{2}, \quad \tilde{\mathbf{F}}_{2}=\mathbf{F}_{1} \\
\tilde{\mathbf{s}}_{1} & =\mathbf{s}_{2}, \tilde{\mathbf{s}}_{2}=\mathbf{s}_{1} .
\end{aligned}
$$

It is assumed here that the perfect channel state information (CSI) is available at the transmitter, therefore the inter cable FEXT is completely canceled by appropriately selecting the modulation matrix F. The inter and intra cable FEXT can be completely canceled by applying conventional precoding schemes, however, this is a sub-optimal solution since it is designed for non-cooperating DAUs. For the system where multiple lines are terminated at each DAU, the optimal solution can be achieved by block diagonalize the matrix HF, where only inter cable FEXT is canceled while the processing is coordinated for the lines terminated at the same DAU. The inter cable FEXT at $\mathrm{RRU}_{k}$ can be completely canceled by imposing the constraint $\mathbf{H}_{k} \mathbf{F}_{m}=0$ for $\forall m \neq k$. The zero forcing constraint can be fulfilled if $\mathbf{F}_{m}$ lies in the null space of $\tilde{\mathbf{H}}_{k}$, where $\tilde{\mathbf{H}}_{k}$ for RRU $\mathrm{RU}_{1}$ and $\mathrm{RRU}_{2}$ are defined as: $\tilde{\mathbf{H}}_{1}=\mathbf{H}_{2}$ and $\tilde{\mathbf{H}}_{2}=\mathbf{H}_{1}$, respectively.

The singular value decomposition (SVD) of the complementary channel matrix $\tilde{\mathbf{H}}_{k}$ can be defined as

$$
\tilde{\mathbf{H}}_{k}=\tilde{\mathbf{U}}_{k} \tilde{\boldsymbol{\Sigma}}_{k}\left[\begin{array}{cc}
\tilde{\mathbf{V}}_{k}^{(1)} & \tilde{\mathbf{V}}_{k}^{(0)}
\end{array}\right]^{*},
$$

where $\tilde{\mathbf{V}}_{k}^{(1)}$ and $\tilde{\mathbf{V}}_{k}^{(0)}$ contain first and last $N$ right singular vectors, respectively. Since $\tilde{\mathbf{V}}_{k}^{(0)}$ provides the orthogonal basis for the null space of complementary channel matrix $\tilde{\mathbf{H}}_{k}$, therefore the columns of $\tilde{\mathbf{V}}_{k}^{(0)}$ can be conveniently used to block diagonalize the overall system. The effective channel matrix after block diagonalization can be written as

$$
\mathbf{H M}=\left[\begin{array}{cc}
\mathbf{H}_{1} \mathbf{M}_{1} & \mathbf{H}_{1} \mathbf{M}_{2} \sim 0 \\
\mathbf{H}_{2} \mathbf{M}_{1} \sim 0 & \mathbf{H}_{2} \mathbf{M}_{2}
\end{array}\right],
$$

where $\mathbf{M}_{k}=\tilde{\mathbf{V}}_{k}^{(0)}$ and $\mathbf{H}_{k} \mathbf{M}_{k}$ is the effective channel matrix for $k$ th DAU. Once the system is block diagonalized by applying zero-forcing constraint, conventional SVD can be used to maximize the capacity at each DAU

$$
\mathbf{H}_{k} \mathbf{M}_{k}=\mathbf{U}_{k} \boldsymbol{\Sigma}_{k} \mathbf{V}_{k}^{(1)},
$$

where $\boldsymbol{\Sigma}_{k}$ contains the singular values of the effective channel matrix $\mathbf{H}_{k} \mathbf{M}_{k}$, while $\mathbf{V}_{k}^{(1)}$ represents the first $N$ right singular vectors of the effective channel that use to define the precoding matrix for the system in Fig. 20 as

$$
\mathbf{F}=\left[\begin{array}{ll}
\mathbf{M}_{1} \mathbf{V}_{1}^{(1)} & \mathbf{M}_{2} \mathbf{V}_{2}^{(1)}
\end{array}\right]
$$

The transmitted signal power is constrained by the line driver connected to each twisted pair, therefore it is desired to normalize the transmit power by a scaling factor $\beta_{\max }$, that is defined as

$$
\beta_{\max }=\sqrt{\max _{m} \sum_{n=1}^{2 N}|\mathbf{F}[m, n]|^{2}} .
$$

The $N \times 1$ received signal at the $k$ th $\mathrm{DAU}$ is

$$
\mathbf{y}_{k}=\frac{1}{\beta_{\max }} \mathbf{U}_{k} \boldsymbol{\Sigma}_{k} \mathbf{s}_{k}+\mathbf{w}_{k} .
$$

The demodulation filter can be defined at the $k$ th DAU as

$$
\mathbf{D}_{k}=\beta_{\max } \boldsymbol{\Sigma}_{k}^{-1} \mathbf{U}_{k}^{H},
$$

that gives the decision variable at the output of the demodulation filter as

$$
\tilde{\mathbf{y}}_{k}=\mathbf{s}_{k}+\mathbf{D}_{k} \mathbf{w}_{k}
$$

The SNR at the decision variable for $j$ th pair connected to $k$ th DAU is

$$
S N R_{j, k}=\frac{\Sigma_{j, k}^{2}}{\beta_{\text {max }}^{2}} S N R_{\text {input }},
$$

where $\Sigma_{j, k}$ is the $j$ th entry of the diagonal matrix $\Sigma_{k}$ and $S N R_{\text {input }}$ is the transmitted signal SNR.

\section{NGMM CAPACITY ANALYSIS}

This section aims to evaluate the achievable capacity per DAU in 5G new Radio (NR) system using a multi-pair cable at the last hop. The NGMM channel environment of two parallel 50m CAT-5 cables (Section IV) that are mutually interfered with each other is considered here for the capacity analysis. The DL precoding based on block diagonalization is then used to precode the NGMM and NGMSM channels as detailed in Section VI. The SNR at the decision variable for $k$ th DAU is computed as in (19), where SNR $_{\text {input }}$ is the transmitted signal SNR that is set to $64 \mathrm{~dB}$. The transmission parameters for the NGMM capacity analysis simulation are summarized in Table I. 


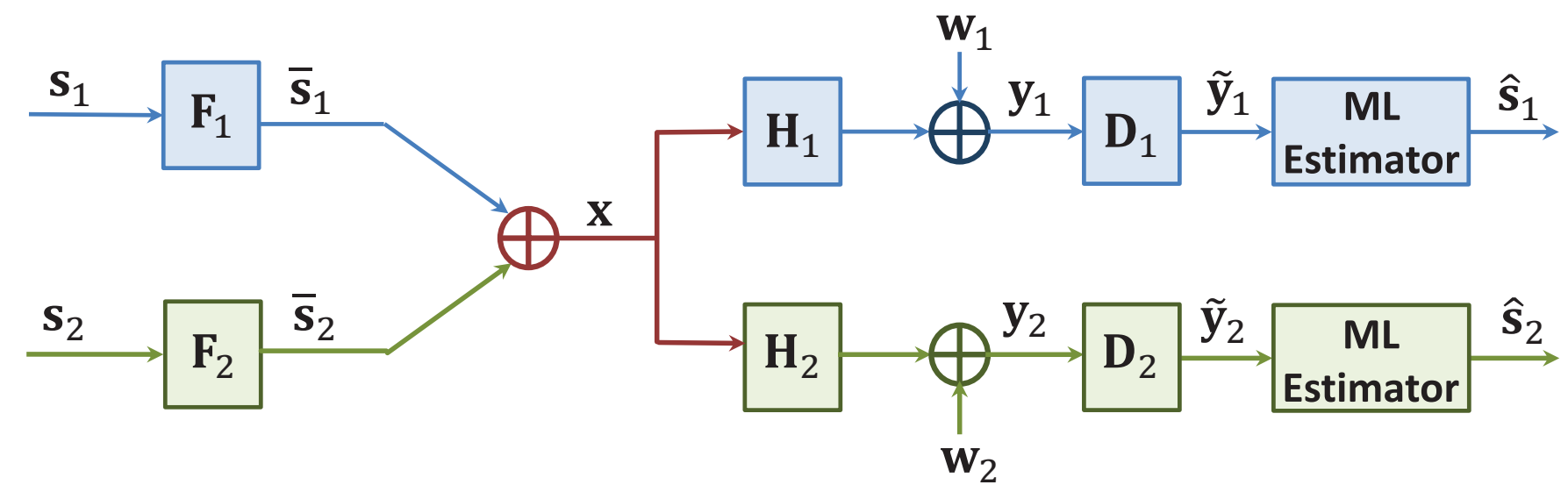

Fig. 20. Block diagram for DL precoding.

TABLE I

TRANSMISSION PARAMETERS FOR NGMM.

\begin{tabular}{|c|c|}
\hline Transmit PSD $\left(P_{S}\right)$ & $-76 \mathrm{dBm} / \mathrm{Hz}$ \\
\hline Background Noise PSD $\left(\boldsymbol{P}_{N}\right)$ & $-140 \mathrm{dBm} / \mathrm{Hz}$ \\
\hline Coding Gain & $5 \mathrm{~dB}$ \\
\hline SNR Margin & $6 \mathrm{~dB}$ \\
\hline SNR Gap & $9.8 \mathrm{~dB}$ \\
\hline Max Spectral efficiency & $16 \mathrm{bps} / \mathrm{Hz}$ \\
\hline Min Spectral efficiency & $2 \mathrm{bps} / \mathrm{Hz}$ \\
\hline Framing overhead & $12 \%$ \\
\hline
\end{tabular}

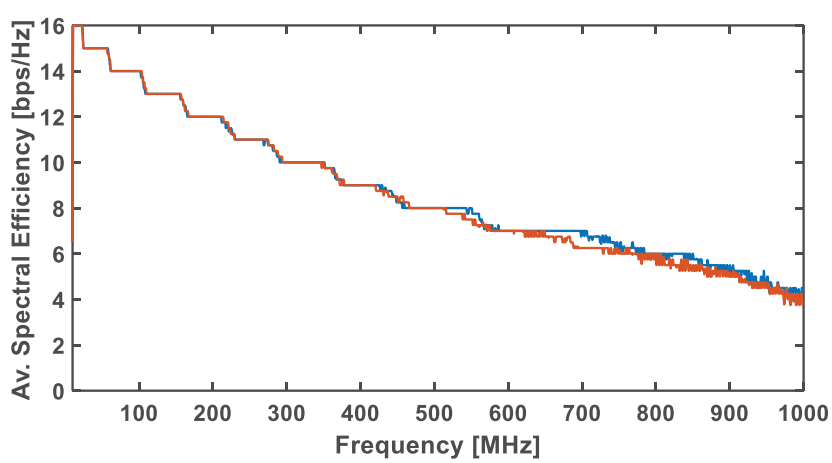

Fig. 21. Average spectral efficiency at $R R U_{1}$ (blue) and $R_{R} U_{2}$ (orange) for NGMM.

Average per line spectral efficiency and sum throughput for $\mathrm{RRU}_{1}$ and $\mathrm{RRU}_{2}$ are examined by considering $1 \mathrm{GHz}$ and 212 $\mathrm{MHz}$ frequency bands for NGMM and NGMSM, respectively. The gap-formula " $(\Gamma[d B]=S N R$ Margin + SNR GapCoding Gain $=10.8 \mathrm{~dB})$ " is used in the simulation setting here to estimate the bit loading over $\ell$ th tone as

$$
b_{\ell}=\log _{2}\left(1+\frac{S N R_{\ell}}{\Gamma}\right)
$$

where $S N R_{\ell}$ is the SNR at the decision variable at $l$ th tone.

The average per line spectral efficiency at $\mathrm{DAU}_{1}$ and $\mathrm{DAU}_{2}$ are shown in Fig. 21 and Fig. 22 for NGMM and NGMSM. The channel bandwidth is extended to $1 \mathrm{GHz}$ in NGMM, however, it is restricted to $212 \mathrm{MHz}$ for NGMSM scenario due to the unavailability of high-frequency transformers that are used to design Phantom mode circuit. The simulation

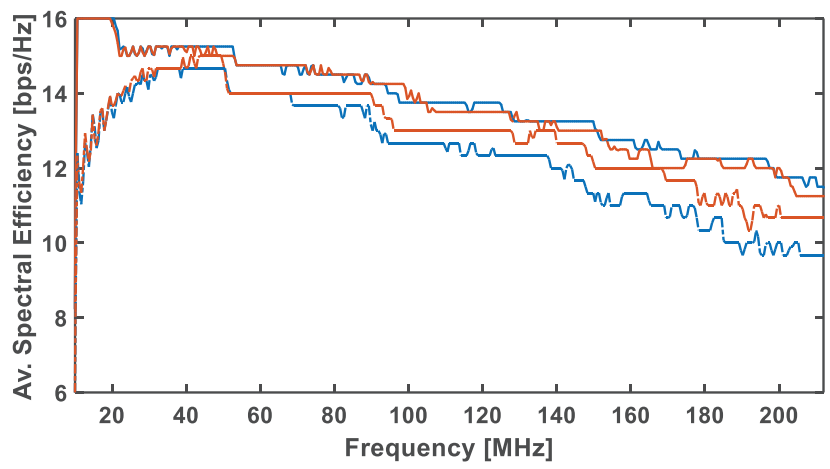

Fig. 22. Average spectral efficiency at $R^{2} U_{1}$ (blue) and $R_{R U}$ (orange) for DM (solid) and PM (dot-dashed) in NGMSM.

settings for multi-pair cable in 5G NR are not yet standardized, therefore the maximum bit loading is set to 16 bits that is achieved over high SNR frequency band in NGMM and only with DM in NGMSM. The CAT-5 cable provides better noise immunity than copper cables use in conventional telephone cabling system, that results in better spectral efficiency even at $1 \mathrm{GHz}$ frequency in NGMM, where the minimum bit loading is 4 bits at $50 \mathrm{~m}$ distance. However the channel characteristics of PM are not good as DM in NGMSM, the minimum bit loading still reaches 10 bits with PM at $212 \mathrm{MHz}$.

The sum throughput at $\mathrm{DAU}_{1}$ and $\mathrm{DAU}_{2}$ of all the pairs over each frequency tone are shown in Fig. 23 and 24 for NGMM and NGMSM, respectively. The sum throughput of the DM and PM pairs are shown separately in Fig. 24 for NGMSM, where the sum capacity for PM is relatively smaller than that is achieved by DM. The difference in sum capacity for PM and DM is because i) the channel characteristics of DM pairs are better than PM pairs, and ii) the number of DM pairs (i.e. 4 pairs) is higher than PM pairs (i.e. 3 pairs). The sum capacity at $\mathrm{DAU}_{1}$ and $\mathrm{DAU}_{2}$ in NGMM is 30.4541 Gbps and 29.9913 Gbps, respectively, over $1 \mathrm{GHz}$ frequency band. In NGMSM, the sum throughput achieved at each DAU by DM and PM is approximately $10 \mathrm{Gbps}$ and $7 \mathrm{Gbps}$, respectively, and it results in overall $17 \mathrm{Gbps}$ per DAU over $212 \mathrm{MHz}$ frequency band. 


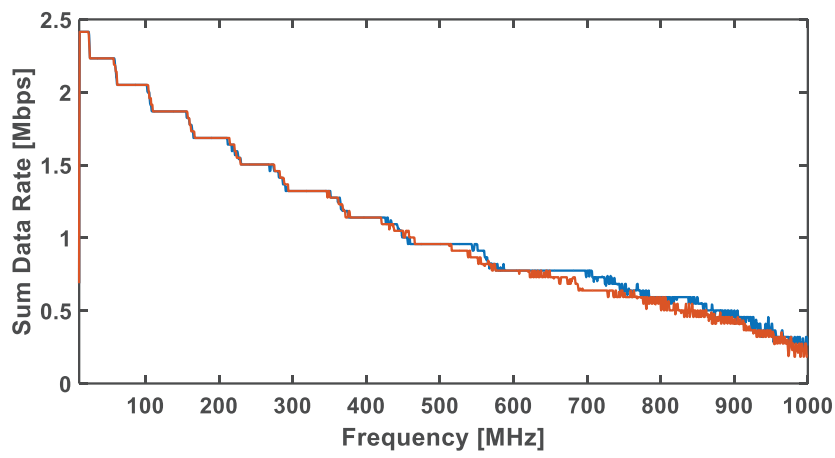

Fig. 23. Sum throughput at $\mathrm{DAU}_{1}$ (blue) and $\mathrm{DAU}_{2}$ (orange) for NGMM.

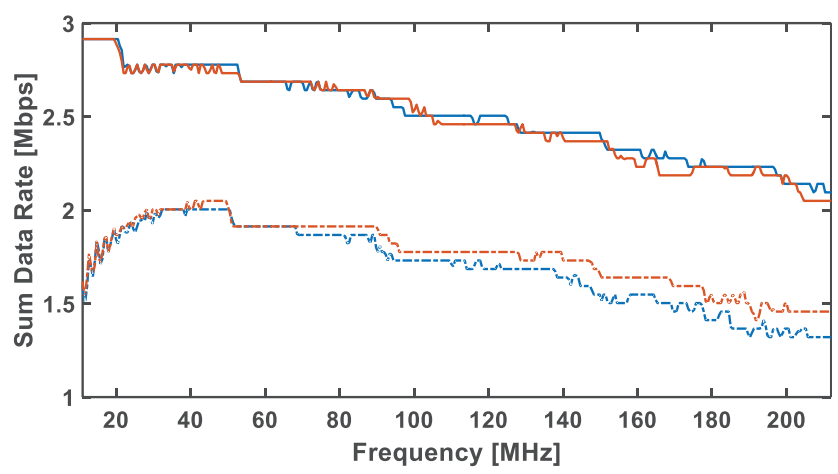

Fig. 24. Sum throughput at $\mathrm{DAU}_{1}$ (blue) and $\mathrm{DAU}_{2}$ (orange) for DM (solid) and PM (dot-dashed) in NGMSM.

\section{CONCLUSION}

This paper aims to provide deep insight details of the measurement and performance of NGMM and NGMSM for front hauling in 5G NR. Methods and apparatus for different variants of NGMM and NGMSM are described in detail with the help of a simple two 50m CAT-5 cable system. The channel measurement results for the test scenario show that the multipair channel is always diagonal dominant in NGMM even at higher frequencies. The diagonal dominant condition is still valid in NGMSM but only for DM pairs since the signal leakage is strong in PM pairs. The measurement results for NGMSM is limited to $225 \mathrm{MHz}$ due to the unavailability of high-frequency transformers for Phantom mode circuits. The system model for DL transmission with 2 DAUs is considered in this paper to evaluate the performance of NGMM and NGMSM system in terms of data rate per DAU. The SVD based block diagonalization is applied at the transmitter for channel precoding such that inter cable FEXT can be mitigated. Since the coordination is possible at the receiver for the cable pairs connected to the same DAU, SVD is individually applied to each DAU considering the effective channel matrix, which in turn maximizes the sum throughput per DAU. The capacity analysis shows that the bandwidth can be extended to $1 \mathrm{GHz}$ in NGMM for a small distance, however, the cable length can be increased in case the bandwidth is not allowed to increase to $\mathrm{GHz}$ range.

\section{REFERENCES}

[1] "New ITU broadband standard fast-tracks route to 1Gbit/s," ITU-T, Dec. 2013.

[2] T. Starr, "Accelerating copper up to a Gigabit in the Broadband Forum," tech. rep., G.fast Summit 2014. Broadband Forum, May 2014.

[3] "Fast access to subscriber terminals (G.fast) - Power spectral density specification," G.9700 ITU-T Rec, April 2014.

[4] "Fast Access to Subscriber Terminals (FAST) - Physical layer specification," G.9701 ITU-T Rec, Dec. 2014.

[5] J. Gambini and U. Spagnolini, "Wireless over cable for femtocell systems," IEEE Communications Magazine, vol. 51, no. 5, pp. 178-185, 2013.

[6] S. H. R. Naqvi, A. Matera, L. Combi, and U. Spagnolini, "On the transport capability of LAN cables in all-analog MIMO-RoC fronthaul," in 2017 IEEE Wireless Communications and Networking Conference (WCNC), pp. 1-6, IEEE, 2017.

[7] S. H. R. Naqvi, P. H. Ho, and S. Jabeen, "A Novel Distributed Antenna Access Architecture for 5G Indoor Service Provisioning," IEEE Journal on Selected Areas in Communications, vol. 36, no. 11, pp. 2518-2527, 2018.

[8] F. Boccardi, R. W. Heath Jr, A. Lozano, T. L. Marzetta, and P. Popovski, "Five disruptive technology directions for 5G," arXiv preprint arXiv:1312.0229, 2013.

[9] A. Checko, H. L. Christiansen, Y. Yan, L. Scolari, G. Kardaras, M. S. Berger, and L. Dittmann, "Cloud ran for mobile networks-A technology overview," IEEE Communications surveys \& tutorials, vol. 17, no. 1, pp. 405-426, 2014.

[10] "Test procedures for digital subscriber line (DSL) transceivers," G.996.1 ITU-T Rec, Feb. 2001.

[11] "Very high speed digital subscriber line transceivers," G.993.1 ITU-T Rec, June 2006.

[12] ETSI TS 101 271, V1.1.1, "Access Terminals Transmission and Multiplexing (ATTM); Access transmission system on metallic pairs; Very High Speed digital subscriber line system (VDSL2)," G.993.1 ITU-T Rec, Jan. 2009.

[13] R. Cendrillon, G. Ginis, E. Van den Bogaert, and M. Moonen, "A nearoptimal linear crosstalk canceler for upstream VDSL," IEEE Transactions on Signal Processing, vol. 54, no. 8, pp. 3136-3146, 2006.

[14] R. Cendrillon, G. Ginis, and M. Moonen, "A near-optimal linear crosstalk precoder for downstream VDSL," IEEE Transactions on Communications, vol. 55, no. 5, pp. 860-863, 2007.

[15] M. Baldi, F. Chiaraluce, R. Garello, M. Polano, and M. Valentini, "Simple statistical analysis of the impact of some nonidealities in downstream VDSL with linear precoding," EURASIP Journal on Advances in Signal Processing, vol. 2010, p. 90, 2010.

[16] D. Acatauassu, S. Höst, C. Lu, M. Berg, A. Klautau, and P. O. Börjesson, "Simple and causal copper cable model suitable for G.Fast frequencies," IEEE Transactions on Communications, vol. 62, no. 11, pp. 4040-4051, 2014.

[17] D. Acatauassu and J. C. Costa, "Refining worst-case crosstalk models for the next generation broadband over copper," in 2017 SBMO/IEEE MTT$S$ International Microwave and Optoelectronics Conference (IMOC), pp. 1-4, IEEE, 2017.

[18] D. Gomes, G. Guedes, A. Klautau, E. Pelaes, and C. Lu, "DSL Phantom Mode Transmission: Cable Measurements and Performance Evaluation," in 4th IEEE Latin-American Conference on Communications, Cuenca, Ecuador, 2012.

[19] C. J. Collins and J. R. Bray, "In situ crosstalk measurements of long cables-the multi-network analyzer method," in 2017 IEEE International Symposium on Electromagnetic Compatibility \& Signal/Power Integrity (EMCSI), pp. 339-344, IEEE, 2017.

[20] Q. H. Spencer, A. L. Swindlehurst, and M. Haardt, "Zero forcing methods for downlink spatial multiplexing in multiuser MIMO channels," IEEE Transactions on Signal Processing, vol. 52, no. 2, pp. 461-471, 2004.

[21] G. Primolevo, O. Simeone, and U. Spagnolini, "Channel aware scheduling for broadcast MIMO systems with orthogonal linear precoding and fairness constraints," in IEEE International Conference on Communications, 2005. ICC 2005. 2005, vol. 4, pp. 2749-2753, IEEE, 2005. 


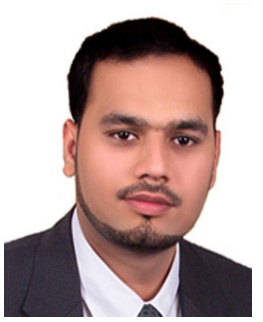

Syed Hassan Raza Naqvi received the B.S. degree in Electronics Engg. from SSUET Pakistan, the M.S. (Electrical Engg.) from Linkoping University, Sweden, and the Ph.D. (IT - specialization in Telecommunication) from Politecnico di Milano, Italy. He is currently Post Doctorate Fellow at Department of Electrical and Computer Engineering, University of Waterloo Canada. He has several years of teaching, research and industrial experience. His research interests include interference mitigation in modern wired and wireless technology, channel estimation and equalization, optimize design and implementation of LTE over cable based front hauling, Massive multi-user MIMO, VLSI and FPGA implementation of modern wired and wireless standards.

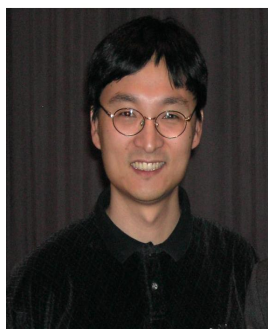

Pin Han Ho received his Ph.D. degree from Queens University at 2002. He is now a full professor in the department of Electrical and Computer Engineering, University of Waterloo, Canada. Pin-Han Ho is the author/coauthor of more than 350 refereed technical papers, several book chapters, and two books on optical networking and survivability. His current research interests cover a wide range of topics in broadband wired and wireless communication networks. He is the recipient of Distinguished Research Excellent Award in the ECE department of UoW, Early Researcher Award in 2005, the Best Paper Award in SPECTS'02, ICC'05, and ICC'07, and the outstanding Paper Award in HPSR02. 